\title{
Zernike Phase Contrast Cryo-electron Tomography of Flagellar Hook-basal Bodies from Vibrio alginolyticus
}

Naoki Hosogi,* Hideki Shigematsu,* Hiroyuki Terashima, ** Michio Homma, ** and Kuniaki Nagayama*

* Department of Strategic Methodology, Okazaki Institute for Integrative Bioscience, 5-1 Higashiyama, Myodaiji-cho, Okazaki, Aichi, 444-8787, Japan

** Graduate School of Science, Nagoya University, Japan

Vibrio alginolyticus swims by using flagella, which play a role of a rotary machine like a corkscrew. The flagellar motor uses the electrochemical potential of sodium ions across the cytoplasmic membrane as the energy source [1]. One single flagellum consists of the filament, the hook and the basal body. The basal body comprising rotor and bushing is constituted of one rod and several structures [2].

In this study, we investigated the structure of the T ring which is a component unique to the sodium ion-driven flagellar motor of $V$. alginolyticus. Using Zernike phase-contrast (ZPC) cryo-electron tomography, 3D structures of the purified hook-basal body (HBB) from a wild type strain and deletion mutant of MotX and MotY ( $\Delta \operatorname{mot} X \Delta m o t Y)$, which are thought to be responsible for the $\mathrm{T}$ ring, have been determined and compared. ZPC electron microscopy enables to take high-contrast images in focus without staining [3]. The phase-retarding plate made of thin carbon film with a hole in the center is positioned in the back-focal plane of the objective lens. The plate can retard the phase of electron waves by half $\pi$, except at the centre where a tiny hole is drilled. Thus we use the inner potential of a thin film as a quarter-wave plate for the scattered electrons to selectively apply half $\pi$ phase shift to the unscattered electrons. So the contrast transfer function (CTF) changes from a sine function to a cosine function. The recovery of low frequency components with the cosine-type CTF can improve the image contrast. Due to the in-focus high-contrast feature of $\mathrm{ZPC}$ electron microscopy, ZPC images of HBB were highly improved in the signal-to-noise ratio, compared to the conventional phase contrast images (FIG. 1.). We could observe the overview of $\mathrm{HBB}$, and find the $\mathrm{T}$ ring only in the HBB from a wild type strain (FIG. 2.). The T ring structure exists beneath the LP ring and seems to form ring-shape structure (FIG. 2.). In contrast, $\mathrm{T}$ ring was lost in the basal body from a $\triangle m o t X \triangle m o t Y$ strain (FIG. 2.). Thus we estimate the $\mathrm{T}$ ring may be comprised of MotX and MotY.

References

[1] T. Yorimitsu and M. Homma, Biochim. Biophys. Acta. 1505 (2001) 82. 
[2] H. Terashima et al., Mol. Microbiol. 62 (2006) 1170.

[3] K. Nagayama and R. Danev, Biophys. Rev. 1 (2009) 37.

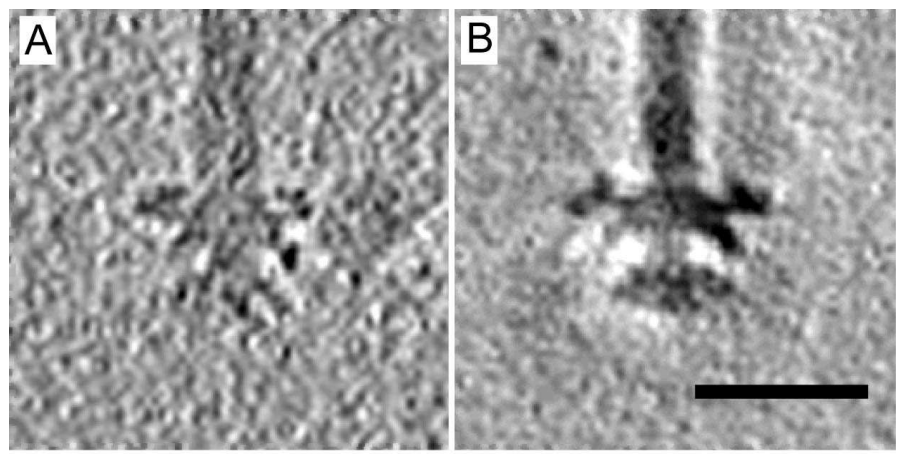

FIG. 1. Conventional and Zernike phase contrast cryo-electron tomograms of vitrified HBB from wild type strain. (A) A $z$ slice of a conventional tomogram. (B) A $z$ slice of a Zernike phase contrast tomogram. $3 \mathrm{~nm}$ thick in $\mathrm{z}$ axis. Scale bar; $50 \mathrm{~nm}$.

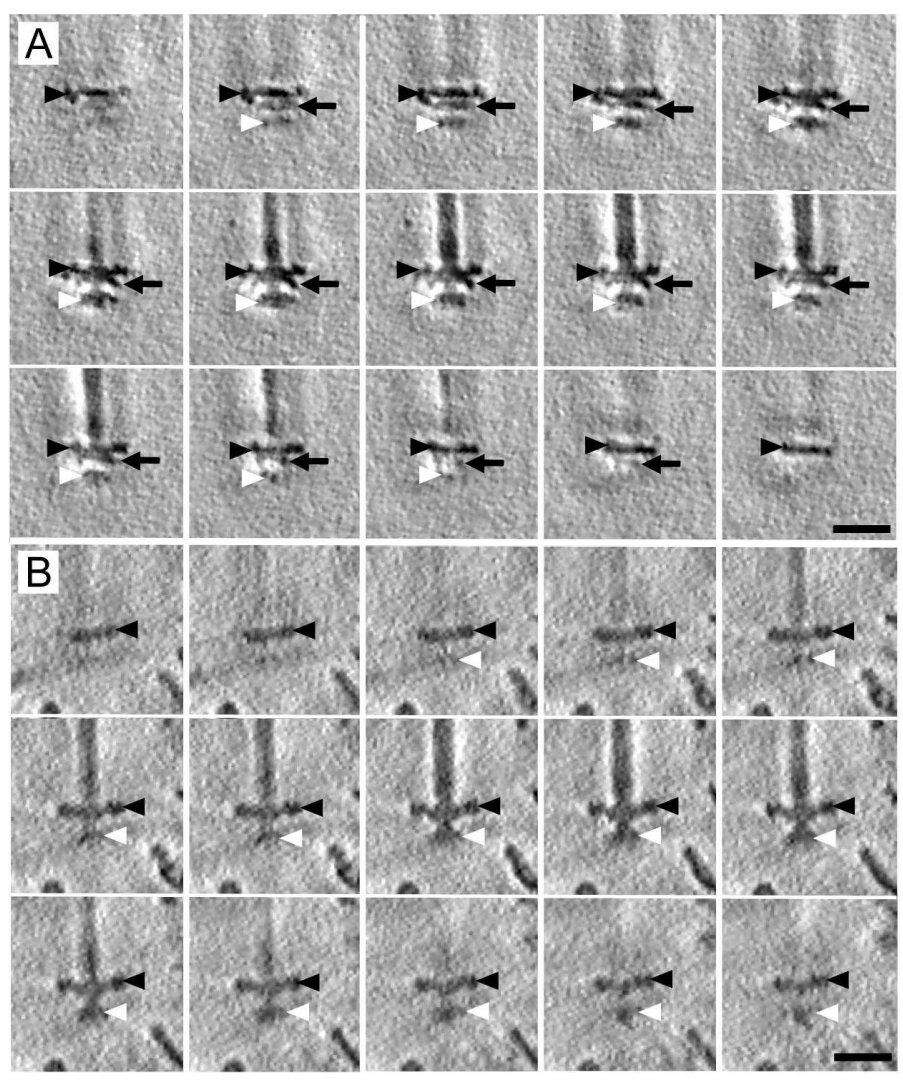

FIG. 2. Serial $z$ slices $(3 \mathrm{~nm}$ thick) through one of the HBB cryo-tomograms. The HBB was purified from a wild type strain (A) and a $\triangle m o t X \triangle m o t Y$ strain (B). Black arrowheads represent LP ring, black arrows represent T ring and white arrowheads represent MS ring. Scale bar=50 nm. 